\title{
Multichannel Control Systems for the Attenuation of Interior Road Noise in Vehicles
}

\author{
Jordan Cheer ${ }^{a}$ and Stephen J. Elliott ${ }^{b}$ \\ Institute of Sound and Vibration Research, \\ University of Southampton, \\ Southampton, \\ Hampshire, SO17 1BJ, \\ U.K.
}

a) corresponding author: j.cheer@soton.ac.uk, +44 2380594934

b) s.j.elliott@soton.ac.uk, +44 2380592384

January 14, 2015 


\begin{abstract}
This paper considers the active control of road noise in vehicles, using either multichannel feedback control, with both headrest and floor positioned microphones providing feedback error signals, or multichannel feedforward control, in which reference signals are provided by the microphones on the vehicle floor and error signals are provided by the microphones mounted on the headrests. The formulation of these control problems is shown to be similar if the constraints of robust stability, limited disturbance enhancement and open-loop stability are imposed. A novel formulation is presented for disturbance enhancement in multichannel systems, which limits the maximum enhancement of each individual error signal. The performance of these two systems is predicted using plant responses and disturbance signals measured in a small city car. The reduction in the sum of the squared pressure signals at the four error microphones for both systems is found to be up to $8 \mathrm{~dB}$ at low frequencies and $3 \mathrm{~dB}$ on average, where the sound level is particularly high from 80 to $180 \mathrm{~Hz}$. The performance of both systems is found to be robust to measured variations in the plant responses. The enhancements in the disturbance at higher frequencies are smaller for the feedback controller than for the feedforward controller, although the performance of the feedback controller is more significantly reduced by the introduction of additional delay in the plant response.
\end{abstract}




\section{Keywords}

Active Noise Control; Road Noise; Feedforward Control; Feedback Control; MIMO

\section{Introduction}

The application of active control methods to reduce the interior noise in road vehicles has been investigated within the automotive industry for around 20 years [1]. Feedforward control of engine noise was first demonstrated in the late 1980s [2] and has since been used in various configurations to control the increase in noise level due to lightweight vehicle design [1], to reduce the variation in the engine noise characteristic due to the use of economical engine designs such as variable displacement [3], and to improve the perceived sound quality of the engine noise [4]. A cost effective feedforward engine noise control system can be implemented using an engine speed reference sensor, low-cost microphone error sensors, the car audio loudspeakers and their amplifiers as control sources and the car audio digital signal processing (DSP) capabilities. Such active engine noise control systems have been implemented by a number of manufacturers.

The design of lightweight vehicles also results in an increase in the low frequency broadband noise, in the car cabin, due to the interaction of the tyres and the road. Feedforward active noise control systems have previously been developed to reduce the noise levels in the car cabin using reference signals obtained by direct measurement of the vibration due to road excitation $[5,6,7]$. Although these feedforward road noise control systems could again be implemented using low-cost microphone error sensors, the car audio loudspeakers as control sources and the car audio DSP capabilities, it is also necessary to employ at least six accelerometers mounted to the vehicle's suspension and bodywork to obtain reference signals with sufficient coherence with the error signals in order to obtain reasonable levels of control [5, 7]. Therefore, such control systems are relatively expensive to implement and have seen limited commercial implementation.

To reduce the cost of implementing a feedforward road noise control system, Mohammad et al [8] have suggested that the necessary reference signals could be obtained using low-cost microphones positioned on the floor of the car cabin. This work showed that similar levels of control could be achievable using reference signals obtained from either accelerometers or microphones, although it was assumed that the acoustic feedback path between the control loudspeakers and the reference microphones is perfectly cancelled and the control filters were not causally constrained. The lack of causality constraint in this work means that the predicted levels of control do not consider the time advance provided by the microphone reference signals compared to the accelerometer reference signals. The effect of the inherent causality constraint on the achievable control must be investigated to determine the suitability of microphone reference signals for road noise cancellation. Additionally, in practice, perfect feedback path cancellation would not be possible, due to variations in the plant response, and it would be necessary to design the controller to be robustly stable to these variations.

An alternative method of reducing the cost of road noise control systems is to use a feedback control architecture. Feedback control of road noise using a single-input single-output controller has already been implemented in a mass-production estate car [9], although significant levels of attenuation are only achieved over a narrowband acoustic resonance, at about $40 \mathrm{~Hz}$, in the front seats. This corresponds to a 
reduction in the first longitudinal enclosure mode. Other modal feedback control systems have also been proposed that may be suitable for similar road noise control problems $[10,11,12]$. To achieve control in vehicles where a single acoustic mode does not dominate the response, however, it has been shown to be necessary to employ a multi-input, multi-output (MIMO) controller [12]. A MIMO feedback controller has been investigated in [13] which uses four headrest mounted microphones as error sensors and the four car audio loudspeakers as control sources. This control system is predicted to achieve an average reduction in road noise at the headrest microphones between 80 and $185 \mathrm{~Hz}$ of around $3 \mathrm{~dB}$, but also increases the average level of the road noise at all microphones by up to $5 \mathrm{~dB}$ between 185 and $240 \mathrm{~Hz}$. The effect of additional delay in the plant response due to a digital implementation of this system has not been considered in this work, and it is expected that this will significantly limit the performance of this feedback control system, as discussed in [14].

This paper considers the design of a multichannel road noise control system employing loudspeakers as control sources, a number of microphones positioned near to the car cabin headrests as error sensors, as in [13], and an additional set of microphones, positioned close to the car cabin floor as reference sensors, to provide additional time advanced information to the controller. This control setup extends the field of active control of road noise in vehicles by fully investigating the limitations of control when microphones are used as the inputs to the controller without the additional feedforward reference signals usually provided by accelerometers mounted to the structure of the vehicle. It is also shown that such a control system can be formulated as either a feedforward, as in [8], or a feedback controller and this paper presents the first comparison of these two formulations in the context of a road noise control system using only microphone input signals.

Section 2 first shows that the proposed control system can be formulated using an equivalent feedback system. For a practical implementation it is necessary to enforce constraints on the robust stability, disturbance enhancement and open-loop controller stability and these design requirements are presented for this multichannel feedback system. A novel formulation of the disturbance enhancement constraint is presented, which limits the enhancement at each individual microphone, instead of the average level at all microphones. It is then shown in Section 2 that the same set of sources and microphones may alternatively be employed in a feedforward control configuration in which the microphones near to the headrests are used as error sensors and the microphones close to the floor are used as reference sensors. Due to the feedback from the control sources to the reference sensors, this feedforward configuration still requires similar constraints to the multichannel feedback controller and these design requirements are presented. Both the feedback and feedforward control strategies presented lead to quadratic cost functions with affine constraints and Section 3 describes a method by which such controllers may be optimised. In Section 4 the performance of the feedforward and feedback control systems are compared in a practical road noise control problem. Finally, Section 5 presents the conclusions of this work.

\section{Multichannel Active Noise Controllers}

\subsection{Feedback Control Employing Internal Model Control Architecture}

The aim of a car cabin road noise control system is to reduce the sound pressure level at the occupants' head positions. Therefore, the proposed multichannel road noise control system uses a number of, $L_{e}$, 


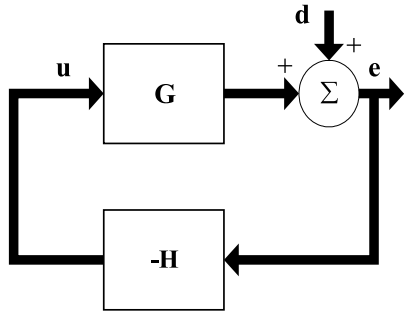

(a) MIMO feedback controller.

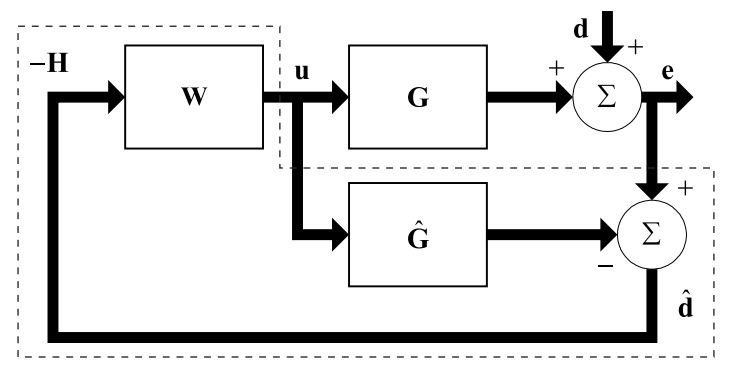

(b) MIMO IMC feedback controller.

Figure 1: Block diagrams of the MIMO (a) and IMC MIMO (b) feedback controllers.

error microphones positioned at the headrest locations, which may also be used in an active feedforward engine noise control system as described in [13]. However, to provide additional information to the feedback controller and improve the performance of the system, the proposed low-cost implementation will also use $K$ microphones positioned on the floor of the car cabin, as previously investigated in [8] for a feedforward formulation with no causality constraint. The total number of error sensors in the multichannel feedback system is $L=L_{e}+K$, such that the $(L \times 1)$ vector of error signals is given by the concatenation of the $\left(L_{e} \times 1\right)$ vector of headrest microphone signals, $\mathbf{e}_{e}$, and the $(K \times 1)$ vector of floor positioned microphone signals, $\mathbf{x}_{e}$; this gives the full vector of errors signals as

$$
\mathbf{e}=\left[\begin{array}{l}
\mathbf{e}_{e} \\
\mathbf{x}_{e}
\end{array}\right] .
$$

To ensure cost-effective implementation, the proposed road noise control system will employ the $M$ lowfrequency car audio loudspeakers as control sources. The multi-input, multi-output (MIMO) feedback structure is shown in Figure 1a. In the frequency domain, the vector of error signals, $\mathbf{e}(j \omega)$, for the MIMO feedback controller can be expressed in terms of the vector of $L$ disturbance signals, $\mathbf{d}(j \omega)$, by

$$
\mathbf{e}(j \omega)=[\mathbf{I}+\mathbf{G}(j \omega) \mathbf{H}(j \omega)]^{-1} \mathbf{d}(j \omega),
$$

where $\mathbf{I}$ is the identity matrix, $\mathbf{H}(j \omega)$ is the $L$ input, $M$ output feedback controller and $\mathbf{G}(j \omega)$ is the matrix of plant responses between the $M$ control sources and $L$ sensors.

Although there is a large number of methods of designing MIMO feedback controllers [15], formulating the MIMO feedback controller using an Internal Model Control (IMC) structure has a number of advantages in the context of active noise control, as discussed in [16], and also provides a framework that allows an interesting comparison between the MIMO feedback controller and the MIMO feedforward controller, considered in the following section. The IMC structure of the MIMO feedback controller is shown in Figure 1b.

The response of the IMC feedback controller shown in Figure 1b, which is contained within the dashed lines, is given by

$$
\mathbf{H}(j \omega)=-[\mathbf{I}+\mathbf{W}(j \omega) \hat{\mathbf{G}}(j \omega)]^{-1} \mathbf{W}(j \omega)
$$

where $\mathbf{W}(j \omega)$ is the frequency response of the $L$ input, $M$ output control filter and $\hat{\mathbf{G}}(j \omega)$ is the matrix of modelled plant responses. If it is assumed that the modelled plant response is perfect then the vector 
of error signals can be written as

$$
\mathbf{e}(j \omega)=[\mathbf{I}+\mathbf{G}(j \omega) \mathbf{W}(j \omega)] \mathbf{d}(j \omega)
$$

and the MIMO controller is then purely feedforward with a vector of reference signals equal to the vector of disturbance signals measured at both the headrest and floor microphones. The feedback controller therefore aims to cancel the disturbance signals using a filtered version of the disturbance signals themselves.

The aim of the multichannel feedback road noise control system is to minimise the sum of the squared error signals corresponding to the $L_{e}$ headrest microphones, which correspond to the first $L_{e}$ elements of the full error vector as detailed in equation 1 . The required cost function for the feedback control system can be expressed as

$$
J(j \omega)=\operatorname{trace}\left[E\left(\mathbf{e}_{e}(j \omega) \mathbf{e}_{e}^{H}(j \omega)\right)\right]
$$

where trace is the sum of the elements on the main diagonal of the square matrix and $E$ is the expectation operator. The vector of error signals at the headrest microphone can be expressed as

$$
\mathbf{e}_{e}(j \omega)=\mathbf{d}_{e}(j \omega)+\mathbf{G}_{e}(j \omega) \mathbf{W}(j \omega) \mathbf{d}(j \omega)
$$

where $\mathbf{d}_{e}(j \omega)$ is the vector of $L_{e}$ disturbance signals measured at the headrest microphones, and $\mathbf{G}_{e}(j \omega)$ is the $\left(L_{e} \times M\right)$ matrix of plant responses between the $M$ control sources and $L_{e}$ headrest error sensors. Substituting this result into equation 5 gives the cost function as

$$
\begin{gathered}
J(j \omega)=\operatorname{trace}\left[\mathbf{G}_{e}(j \omega) \mathbf{W}(j \omega) \mathbf{S}_{d d}(j \omega) \mathbf{W}^{H}(j \omega) \mathbf{G}_{e}^{H}(j \omega)+\mathbf{G}_{e}(j \omega) \mathbf{W}(j \omega) \mathbf{S}_{d d_{e}}^{H}(j \omega)+\cdots\right. \\
\left.\mathbf{S}_{d d_{e}}(j \omega) \mathbf{W}^{H}(j \omega) \mathbf{G}_{e}^{H}(j \omega)+\mathbf{S}_{d_{e} d_{e}}(j \omega)\right]
\end{gathered}
$$

where $\mathbf{S}_{d d}(j \omega)$ is the spectral density matrix of the $L$ disturbance signals, $\mathbf{S}_{d_{e} d_{e}}(j \omega)$ is the spectral density matrix of the $L_{e}$ headrest microphone signals, and $\mathbf{S}_{d d_{e}}(j \omega)$ is the spectral density matrix between the $L$ disturbance signals and $L_{e}$ headrest disturbance signals. It should be highlighted that although the feedback controller attempts to minimise the sum of the squared pressures at the headrest error sensors, $\mathbf{e}_{e}$, the multichannel control filter matrix, $\mathbf{W}$, is driven by the full vector of error signals given by equation 1 and, therefore, has the potential to benefit from the time advanced information provided by the $K$ error sensors positioned on the floor of the car cabin.

In practice the plant model, $\hat{\mathbf{G}}(j \omega)$, used in the IMC feedback controller shown in Figure $1 \mathrm{~b}$ will not be perfect due to modelling inaccuracies and the variation in the physical responses with parameters such as the number of occupants in the car cabin. These inaccuracies will result in a degree of feedback in the system and, therefore, it is necessary to design the feedback controller to be robustly stable to differences between the modelled and physical plant responses.

A robust stability constraint for the MIMO feedback controller can be obtained by assuming that the uncertainty in the plant response can be modelled as multiplicative output uncertainty. Although this assumption has some limitations in the case of a MIMO system [15], it provides a useful starting point in this application. The multiplicative output uncertainty model describes the matrix of feedback path 
responses at any time according to the relationship

$$
\mathbf{G}(j \omega)=\left[\mathbf{I}+\boldsymbol{\Delta}_{O}(j \omega)\right] \mathbf{G}_{0}(j \omega)
$$

where $\mathbf{G}_{0}(j \omega)$ is the matrix of nominal plant responses and $\boldsymbol{\Delta}_{O}(j \omega)$ is a fractional uncertainty matrix, whose maximum singular value at each frequency is bounded by

$$
\bar{\sigma}\left(\boldsymbol{\Delta}_{0}(j \omega)\right) \leq B(j \omega)
$$

where $\bar{\sigma}$ is the maximum singular value and $B(j \omega)$ is a real number describing the bound on the uncertainty. The robust stability of the MIMO IMC feedback controller shown in Figure 1b with multiplicative output uncertainty can be analysed using the $\mathbf{M}-\boldsymbol{\Delta}$ structure, as described in [15, 17], and shown in Figure 2, where the plant model is equal to the nominal plant response, $\hat{\mathbf{G}}=\mathbf{G}_{0}$. According to the general robust stability theorem, if we assume that the nominal system $\mathbf{M}$, contained within the dotted lines in Figure 2, and the plant perturbations, $\boldsymbol{\Delta}$, are stable then the complete system is stable if the Nyquist plot of $\operatorname{det}[\mathbf{I}-\mathbf{M} \boldsymbol{\Delta}]$ does not encircle the origin for all allowed perturbations [17, 15]. This leads to the general robust stability condition [15]

$$
\bar{\sigma}(\mathbf{M}(j \omega)) \bar{\sigma}(\boldsymbol{\Delta}(j \omega))<1 \text { for all } \omega, \boldsymbol{\Delta} \text {. }
$$

From Figure 2 it can be seen that for the MIMO IMC feedback controller with multiplicative output uncertainty, the nominal response, $\mathbf{M}$, is given by

$$
\mathbf{M}(j \omega)=\mathbf{G}_{0}(j \omega) \mathbf{W}(j \omega)
$$

and, therefore, the robust stability condition according to equation 10 is

$$
\bar{\sigma}\left(\mathbf{G}_{0}(j \omega) \mathbf{W}(j \omega)\right) \bar{\sigma}\left(\boldsymbol{\Delta}_{O}(j \omega)\right) \leq 1 \text { for all } \omega, \boldsymbol{\Delta} .
$$

Finally, we can substitute in the upper bound on the output multiplicative plant uncertainty using equation 9 and the robust stability constraint can be expressed as

$$
\bar{\sigma}\left(\mathbf{G}_{0}(j \omega) \mathbf{W}(j \omega)\right) B(j \omega) \leq 1 \text { for all } \omega \text {. }
$$

This robust stability constraint is based on the small gain theorem which is in general somewhat conservative [18] and a tighter bound on the robust stability may be provided by using a less conservative approach, for example $[19,20]$. These less conservative robust stability constraints require specific assumptions to be made about the uncertainty matrix, $\boldsymbol{\Delta}$. For example, Haddad and Bernstein [19] assume that the uncertainty is positive real and detail conditions under which this may be a reasonable assumption. However, in the context of the car cabin control system employing loudspeakers and microphones, there is a large number of unrelated sources of plant uncertainty, such as the number of occupants, the position of opening windows and the temperature, for example, as discussed in [3, 21] and, therefore, it is difficult to reliably make such assumptions about the uncertainty matrix. Under these conditions the direction of the gain in $\boldsymbol{\Delta}$ closely approximates a random function and the robust stability constraint 


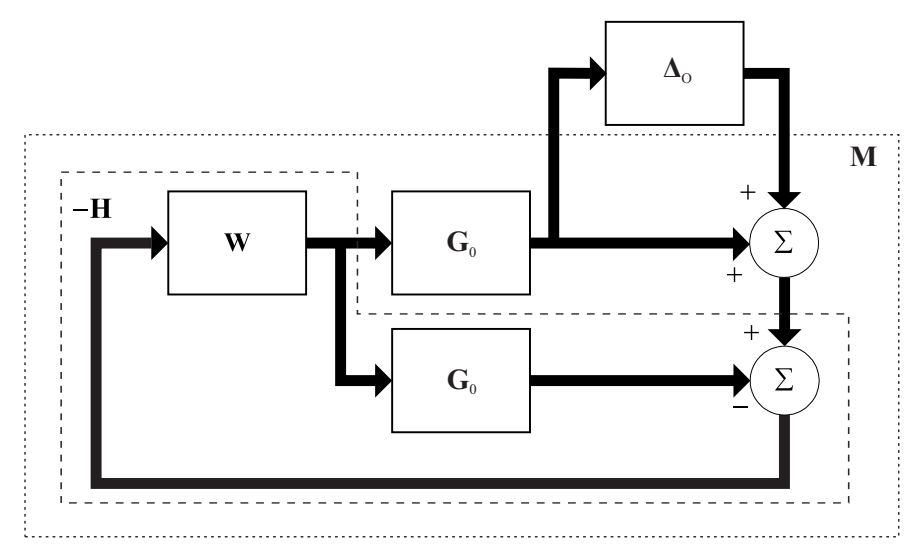

Figure 2: MIMO IMC feedback controller with output uncertainty in the plant response, arranged in the M- $\Delta$ form.

based on the small gain theorem provides a reasonable bound on the robust stability. Consequently, we expect the performance of the control systems designed here to be similar to what may be achieved in a commercial automotive implementation.

In active noise control it is important that the controller does not achieve high levels of attenuation at the expense of producing high levels of enhancement outside of the control bandwidth. This can be achieved by implementing a disturbance enhancement constraint and in the case of the multi-sensor system there are a number of possible formulations for this constraint. For example, it is possible to enforce a constraint on the maximum enhancement in the sum of the squared error signals [16]. A more relevant constraint for this application is introduced here, however, which involves constraining the maximum enhancement in the individual error signals. This will provide a more uniform reduction in the noise, by avoiding high levels of enhancements at some error microphones being balanced out by reductions at other microphones [11]. For the road noise control problem it is the enhancements at the occupant's head positions that are of interest and these are described by the vector of headrest error microphone signals, $\mathbf{e}_{e}(j \omega)$, which corresponds to the first $L_{e}$ elements of the full $L$ error vector $\mathbf{e}(j \omega)$. This constraint on the maximum enhancement in the individual disturbance signals can then be expressed as

$$
\max \left[\operatorname{diag}\left(\mathbf{D}(j \omega) E\left(\mathbf{e}_{e}(j \omega) \mathbf{e}_{e}^{H}(j \omega)\right)\right)\right] \frac{1}{A}<1 \quad \text { for all } \omega
$$

where $\mathbf{D}(j \omega)$ is the $L \times L$ diagonal matrix given by

$$
\mathbf{D}(j \omega)=\left[\begin{array}{cccc}
\frac{1}{E\left|d_{e 1}(j \omega)\right|^{2}} & 0 & 0 & 0 \\
0 & \frac{1}{E\left|d_{e 2}(j \omega)\right|^{2}} & 0 & 0 \\
0 & 0 & \ddots & 0 \\
0 & 0 & 0 & \frac{1}{E\left|d_{e L}(j \omega)\right|^{2}}
\end{array}\right]
$$

where $d_{e l}(j \omega)$ is the disturbance signal measured at the $l$-th headrest error sensor and the maximum enhancement in the $L_{e}$ magnitude squared headrest disturbance signals will be less than a maximum value defined by $A$.

Using the control structure shown in Figure $1 \mathrm{~b}$ it is possible for the optimal controller to be open-loop unstable but closed-loop stable, as previously noted in $[16,17]$. In a practical real-time system, the use 
of an open-loop unstable controller would be dangerous since any temporary disruption of the normal plant response would cause the system to go unstable. Such a controller is also difficult to realise, since it would not be possible to measure the response of the open-loop controller and, therefore, it would be difficult to ensure that the expected closed-loop performance would be achieved. This issue has been discussed in the context of a single-channel active headrest system employing a virtual microphone in [22] and in order to ensure a stable open-loop controller an additional design constraint was proposed that limits the response of the open-loop controller to be on the right hand side of the Nyquist point. For the MIMO feedback controller in Figure 1b a similar constraint can be enforced by constraining the real part of the maximum eigenvalue of the open-loop controller response, that is

$$
-\Re\left(\bar{\lambda}\left(\mathbf{G}_{0}(j \omega) \mathbf{W}(j \omega)\right)\right)<1
$$

where $\bar{\lambda}$ denotes the maximum eigenvalue of the square matrix.

The overall design of the feedback controller shown in Figure 1b can be expressed as minimising the cost function given by equation 5 , which is the sum of the squared signals measured at the $L$ headrest error microphones, subject to the robust stability constraint given by equation 13, the disturbance enhancement constraint given by equation 14 and the open-loop controller stability constraint given by equation 16 .

\subsection{Feedforward Control Employing Feedback Path Cancellation}

The multi-input, multi-output control system employing $L$ microphones and $M$ control sources may also be formulated using a feedforward control structure. In this case the $L_{e}$ signals from the headrest microphones are used as error signals, while the $K$ signals from the microphones positioned on the floor of the car cabin are used as time advanced reference signals. Therefore, the control signals in this case are generated by filtering the $K$ signals from the floor positioned microphones only, rather than the full set of $L=L_{e}+K$ microphones as in the feedback formulation. This controller design has previously been investigated in [8], although in this study it was assumed that the acoustic feedback path was completely cancelled and the presented simulations did not contain the causality of the controller. The standard multi-input, multi-output (MIMO) feedforward controller with $L_{e}$ error sensors, $M$ control sources and $K$ reference sensors is shown in Figure 3a. In practice the feedforward controller is adapted in order to adjust its response to changes in the disturbance and plant response, so that the whole system becomes closed loop [18], but we will concentrate here on the limits of performance without this complexity.

It is assumed, initially, that the output of the reference sensors are unaffected by the output of the control sources, so that the system is entirely feedforward. In the frequency domain the vector of headrest error signals, $\mathbf{e}_{e}(j \omega)$, for the feedforward control system in Figure 3a is given by

$$
\begin{aligned}
\mathbf{e}_{e}(j \omega) & =\mathbf{d}_{e}(j \omega)+\mathbf{G}_{e}(j \omega) \mathbf{u}(j \omega), \\
& =\mathbf{d}_{e}(j \omega)+\mathbf{G}_{e}(j \omega) \mathbf{W}_{x}(j \omega) \mathbf{x}(j \omega),
\end{aligned}
$$

where $\mathbf{d}_{e}(j \omega)$ is the vector of disturbance signals measured at the $L_{e}$ headrest error sensors, $\mathbf{G}_{e}(j \omega)$ is the matrix of plant responses between the $M$ control sources and $L_{e}$ headrest error sensors, $\mathbf{u}(j \omega)$ is the vector of $M$ control signals, $\mathbf{W}_{x}(j \omega)$ is the $M \times K$ matrix of feedforward control filters and $\mathbf{x}(j \omega)$ is 


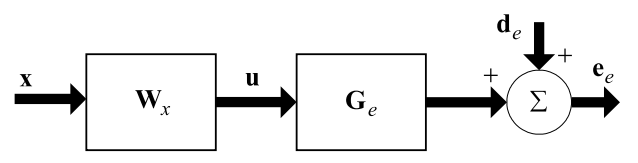

(a) MIMO feedforward controller.

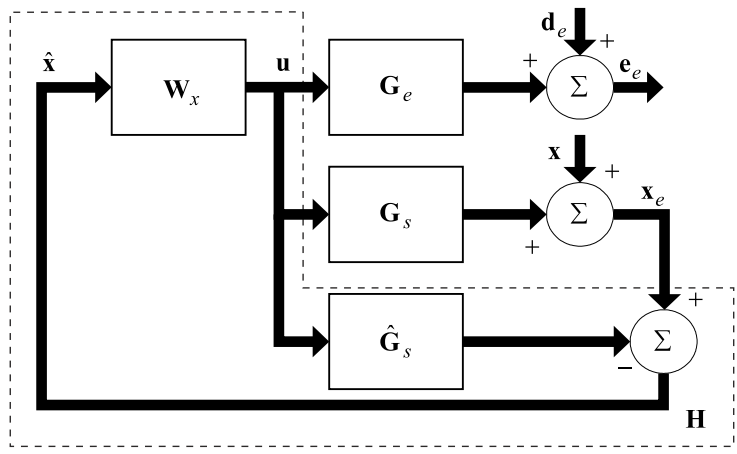

(b) MIMO feedforward controller with feedback cancellation.

Figure 3: Block diagrams of the MIMO (a) and IMC MIMO (b) feedforward controllers.

the vector of reference signals measured at the $K$ reference microphones located on the floor of the car cabin. From equation 18 it can be seen that the feedforward controller attempts to cancel the disturbance signals using filtered reference signals and, therefore, the performance of the controller is dependent on the multiple coherence between the reference and disturbance signals [14].

If the reference signals for the feedforward road noise controller were obtained from non-acoustic sensors, then equation 18 would completely describe the response of the system and may be used to design a suitable controller. However, for the feedforward controller employing microphone reference sensors positioned on the floor of the car cabin, there will be feedback paths between the control sources and the reference microphones via the $K \times M$ matrix of responses $\mathbf{G}_{s}(j \omega)$. These feedback paths may be compensated for by employing an internal model of their responses, $\hat{\mathbf{G}}_{s}(j \omega)$, in the controller as shown in Figure 3b [14]. Such an arrangement was originally inspired by electrical echo cancellers but the operation of the internal plant model can be related to the internal model control architecture discussed in the previous section. The matrix of responses for the complete controller contained within the dashed lines in Figure $3 \mathrm{~b}$ is given by

$$
\mathbf{H}(j \omega)=\left[\mathbf{I}+\mathbf{W}_{x}(j \omega) \hat{\mathbf{G}}_{s}(j \omega)\right]^{-1} \mathbf{W}_{x}(j \omega)
$$

The aim of the internal model of the feedback paths is to cancel out the contribution of the control signals to the signals measured at the reference microphones, which is given by $\mathbf{G}_{s}(j \omega) \mathbf{u}(j \omega)$. In this case the feedforward control filter, $\mathbf{W}_{x}(j \omega)$, is fed with an estimate of the reference signals, $\hat{\mathbf{x}}(j \omega)$, which is given by

$$
\hat{\mathbf{x}}(j \omega)=\mathbf{x}(j \omega)-\left(\hat{\mathbf{G}}_{s}(j \omega)-\mathbf{G}_{s}(j \omega)\right) \mathbf{u}(j \omega)
$$

The vector of error signals for the feedforward controller with feedback cancellation is then given by

$$
\mathbf{e}_{e}(j \omega)=\mathbf{d}_{e}(j \omega)+\mathbf{G}_{e}(j \omega)\left[\mathbf{I}+\left(\hat{\mathbf{G}}_{s}(j \omega)-\mathbf{G}_{s}(j \omega)\right) \mathbf{W}_{x}(j \omega)\right]^{-1} \mathbf{W}_{x}(j \omega) \mathbf{x}(j \omega)
$$

From equation 21 it can be seen that if the feedback path model is equal to the physical feedback path then the system becomes equal to equation 18 and the controller in Figure 3b becomes purely feedforward.

In the case where the feedback from the control sources to the reference sensors is cancelled, the controller can be designed to minimise the sum of the $L_{e}$ squared error signals measured at the headrest 
microphones given by equation 18 . The cost function to minimise is then

$$
\begin{aligned}
J(j \omega)=\operatorname{trace}[ & \left.E\left(\mathbf{e}_{e}(j \omega) \mathbf{e}_{e}^{H}(j \omega)\right)\right] \\
=\operatorname{trace}[ & \mathbf{G}_{e}(j \omega) \mathbf{W}_{x}(j \omega) \mathbf{S}_{x x}(j \omega) \mathbf{W}_{x}^{H}(j \omega) \mathbf{G}_{e}^{H}(j \omega)+\mathbf{G}_{e}(j \omega) \mathbf{W}_{x}(j \omega) \mathbf{S}_{x d_{e}}(j \omega)+\cdots \\
& \left.\mathbf{S}_{x d_{e}}^{H}(j \omega) \mathbf{W}_{x}^{H}(j \omega) \mathbf{G}_{e}^{H}(j \omega)+\mathbf{S}_{d_{e} d_{e}}(j \omega)\right],
\end{aligned}
$$

where $\mathbf{S}_{x x}(j \omega)$ is the spectral density matrix of the reference signals measured at the floor microphones, and $\mathbf{S}_{x d_{e}}(j \omega)$ is the cross spectral density matrix between the reference and disturbance signals measured at the headrest microphones. This cost function is quadratic with respect to the control filter coefficients and the unconstrained, nominal solution can be calculated using standard Wiener methods, as in [8]. In a practical implementation, however, the feedback paths will not be perfectly modelled, as discussed in the context of the MIMO feedback controller in the previous section. These model inaccuracies will result in a degree of feedback in the system and, therefore, it is necessary to design the feedforward controller to be robustly stable to changes in the response of the feedback paths.

A robust stability constraint for the feedforward controller with feedback cancellation can be obtained by again assuming that the uncertainty in the feedback paths can be modelled as multiplicative output uncertainty, as described for the MIMO feedback controller in the previous section. Since both $\mathbf{G}_{s}(j \omega)$ in this section and $\mathbf{G}(j \omega)$ in the previous section are determined by the acoustic field in the car, the multiple sources of uncertainty in $\mathbf{G}_{s}(j \omega)$ will be similar to those discussed above for $\mathbf{G}(j \omega)$, and thus we once again justify the use of the small gain theorem based robust stability constraint in this case. The M- $\boldsymbol{\Delta}$ structure for the MIMO feedforward controller with feedback cancellation is shown in Figure 4 where the plant model is equal to the nominal plant response, $\hat{\mathbf{G}}_{s}(j \omega)=\mathbf{G}_{s}(j \omega)$. It is worth noting that the feedforward component of this system is external to the $\mathbf{M}-\boldsymbol{\Delta}$ structure as it is open-loop and therefore does not affect the system's stability. Following the analysis presented for the feedback controller, the robust stability condition for the feedforward control with feedback path cancellation and a multiplicative output plant uncertainty is given by

$$
\bar{\sigma}\left(\mathbf{G}_{s 0}(j \omega) \mathbf{W}_{x}(j \omega)\right) B(j \omega) \leq 1 \text { for all } \omega .
$$

The disturbance enhancement constraint for the MIMO feedforward controller can be enforced in exactly the same way as that for the feedback controller, since it is still the enhancements in the noise levels at the occupant's head positions that are of interest. This constraint is given by equation 14 .

As in the feedback controller using IMC, it is possible for the optimal feedforward controller with feedback path cancellation to be open-loop unstable but closed-loop stable, although this is impractical as discussed above. Therefore, it is once again necessary to enforce a constraint on the real part of the maximum eigenvalue of the open-loop controller response, which in the MIMO feedforward controller with feedback cancellation is given by

$$
-\Re\left(\bar{\lambda}\left(\mathbf{G}_{s 0}(j \omega) \mathbf{W}_{x}(j \omega)\right)\right)<1 .
$$

The overall design of the feedforward controller shown in Figure $3 \mathrm{~b}$ can then be expressed as minimis- 


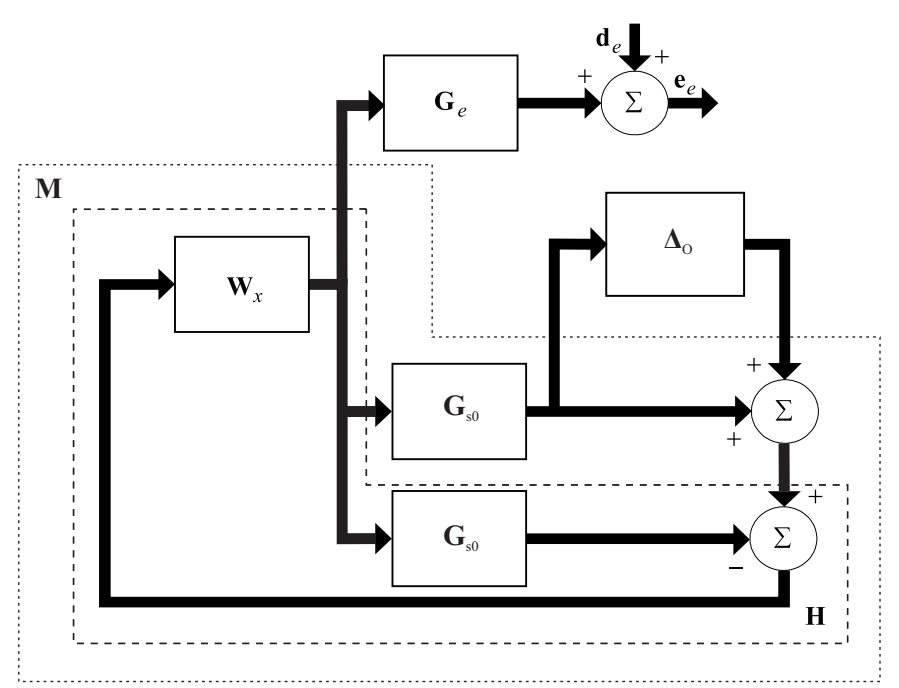

Figure 4: MIMO feedforward controller with feedback cancellation path with output uncertainty in the feedback plant response, arranged in the $\mathbf{M}-\boldsymbol{\Delta}$ form.

ing the cost function given by equation 22 subject to the robust stability constraint given by equation 24, the disturbance enhancement constraint given by equation 14 and the open-loop controller stability constraint given by equation 25 .

\section{Optimisation of Multichannel Active Noise Controllers for Road Noise Control}

In the previous section it has been shown that the design of both the multichannel feedback and feedforward road noise control systems requires the minimisation of a quadratic cost function subject to a number of constraints. Although it is non-trivial to obtain an exact solution to the continuous frequency domain problem, a near optimal causal controller response can be obtained by representing the control filters as a bank of finite impulse response (FIR) filters of sufficient length and solving the optimisation problem in the discrete frequency domain. This method has previously been applied to the design of a single-input, single-output active noise control system in [16]. The optimisation problem here is convex $[23,24]$, since it involves the minimisation of a quadratic cost function with affine constraints, and hence has a unique global minimum and, as such, it is guaranteed to converge to the single optimal solution. This fact has previously been employed in the context of active noise control [25], active vibration control [24] and more generally in [23].

\subsection{Feedback Controller}

The design of the MIMO feedback controller shown in Figure 1b requires the specification of the control filter matrix $\mathbf{W}$, which filters the $L=L_{e}+K$ microphone signals at the headrest and floor positions to produce the $M$ control signals. If the control filter matrix in this is case is defined as an $M L$ bank of FIR filters, w, each with $I$ coefficients, then the cost function given by equation 5 becomes a quadratic function of the filter coefficients. By discretising the frequency responses at $N$ linearly spaced frequencies, 
$k$, the constrained optimisation can be expressed using equations 7, 13, 14 and 16 as

$$
\begin{aligned}
\min _{\mathbf{w}} & \frac{1}{N} \sum_{k=k_{1}}^{k_{2}} \operatorname{trace}\left[\mathbf{G}_{e}(k) \mathbf{W}(k) \mathbf{S}_{d d}(k) \mathbf{W}^{H}(k) \mathbf{G}_{e}^{H}(k)+\cdots\right. \\
& \left.\mathbf{G}_{e}(k) \mathbf{W}(k) \mathbf{S}_{d d_{e}}^{H}(k)+\mathbf{S}_{d d_{e}}(k) \mathbf{W}^{H}(k) \mathbf{G}_{e}^{H}(k)+\mathbf{S}_{d_{e} d_{e}}(k)\right] \\
\text { subject to } \quad & \bar{\sigma}\left(\mathbf{G}_{0}(k) \mathbf{W}(k)\right) B(k)<1 \quad \text { for all } k, \\
& \max \left[\operatorname{diag}\left(\mathbf{D}(k) E\left(\mathbf{e}_{e}(k) \mathbf{e}_{e}^{H}(k)\right)\right)\right] \frac{1}{A}<1 \quad \text { for all } k, \\
& -\Re\left(\bar{\lambda}\left(\mathbf{G}_{0}(k) \mathbf{W}(k)\right)\right)<1 \quad \text { for all } k,
\end{aligned}
$$

where $k_{1}$ and $k_{2}$ define the lower and upper bounds over which disturbance attenuation is desired. Since the cost function is quadratic with respect to the filter coefficients and the constraints are affine functions of the filter coefficients, the optimal solution can be obtained using sequential quadratic programming [23], although alternative programming methods could also be employed [16]. It is important to highlight that the constrained optimisation given by equation 26 directly calculates the vector w of $M L I$ FIR filter coefficients. At each iteration the frequency responses of the $M L$ control filters, $\mathbf{W}$, are calculated from the FIR filter responses at the $N$ linearly spaced frequencies using the Matlab function freqz, which uses Horner's method of nested polynomial evaluation [26]; the cost function and constraints given in equation 26 are then calculated in the frequency domain in order for the optimisation to calculate the subsequent iteration of the optimisation process. So, although only $I$ coefficients per filter are being calculated at each iteration, the frequency domain constraints are being checked at a $N$ frequencies, which may be greater than $I$ to ensure that violations at any frequency are detected.

To ensure that the solution to the discrete problem given by equation 26 approximates the desired solution to the continuous problem it is important that $N$ is large enough such that the discretised frequency responses are accurate representations of the actual responses. This can be achieved by ensuring that the impulse responses of the discretised responses have negligible amplitude at the end of their responses [16]. It is also important to ensure that the FIR control filters are sufficiently long such that the obtained solution is optimal and this can be ensured by gradually increasing the length of $I$ until there is no further improvement in performance [24].

\subsection{Feedforward Controller}

The design of the MIMO feedforward controller with feedback cancellation shown in Figure $3 \mathrm{~b}$ requires the specification of the control filter matrix $\mathbf{W}_{x}$, which in this case filters the $K$ reference signals measured by the microphones located on the floor of the car cabin only to produce the $M$ control signals. If the control filter matrix is defined as an $M K$ bank of FIR filters, $\mathbf{w}_{x}$, each with $I$ coefficients, then the cost function given by equation 22 becomes a quadratic function of the filter coefficients. If all frequency responses are then discretised in the frequency domain at $N$ linearly spaced frequencies, the constrained 
optimisation can be expressed using equations $23,24,14$ and 25 as

$$
\begin{array}{ll}
\min _{\mathbf{w}_{x}} \quad \frac{1}{N} \sum_{k=k_{1}}^{k_{2}} \operatorname{trace}\left[\mathbf{G}_{e}(k) \mathbf{W}_{x}(k) \mathbf{S}_{x x}(k) \mathbf{W}_{x}^{H}(k) \mathbf{G}_{e}^{H}(k) \cdots\right. \\
\left.\quad+\mathbf{G}_{e}(k) \mathbf{W}_{x}(k) \mathbf{S}_{x d_{e}}(k)+\mathbf{S}_{x d_{e}}^{H}(k) \mathbf{W}_{x}^{H}(k) \mathbf{G}_{e}^{H}(k)+\mathbf{S}_{d_{e} d_{e}}(k)\right] \\
\text { subject to } \quad \bar{\sigma}\left(\mathbf{G}_{s 0}(k) \mathbf{W}_{x}(k)\right) B(k)<1 \quad \text { for all } k, \\
\max \left[\operatorname{diag}\left(\mathbf{D}(k) E\left(\mathbf{e}_{e}(k) \mathbf{e}_{e}^{H}(k)\right)\right)\right] \frac{1}{A}<1 \quad \text { for all } k, \\
-\Re\left(\bar{\lambda}\left(\mathbf{G}_{s 0}(k) \mathbf{W}_{x}(k)\right)\right)<1 \quad \text { for all } k,
\end{array}
$$

and this can again be solved using sequential quadratic programming due to the convexity of the optimisation problem. Once again, it is necessary to define the length of the control filters, $I$, and the frequency discretisation resolution, $N$, such that the solution to the optimisation problem approximates the desired continuous frequency solution. As for the feedback controller optimisation, the FIR filter coefficients are directly optimised, while at each iteration their frequency responses are calculated using the Matlab function freqz in order to evaluate the frequency domain cost function and constraints given by equation 27 .

\section{Performance of Multichannel Road Noise Control Systems}

To compare the potential performance of the two methods of designing the multichannel active road noise control system, a system consisting of eight microphones and four loudspeakers has been considered in a small city car. The proposed multichannel active road noise control system consists of two arrays of microphones - one array of four microphones positioned close to the car cabin headrests and one array of four microphones positioned on the floor of the car cabin. Although the microphones employed at both the headrest and floor positions may be of low-cost, they must still have a sufficiently broad bandwidth to monitor the road noise disturbance. The microphones employed in the system presented here are lowcost 1/4 inch electret type microphones, with a bandwidth of $20 \mathrm{~Hz}$ to $16 \mathrm{kHz}$, a sensitivity variation of $\pm 3 \mathrm{~dB}$, and a noise floor that provides at least $40 \mathrm{~dB}$ of signal to noise ratio; this provides sufficient bandwidth for the road noise disturbance and the variations in the response of the microphones are included in the controller design via the plant response model which is determined during an initial plant identification phase. The control sources are provided by the four low-frequency car audio loudspeakers, two of which are positioned in the front doors of the small city car and two of which are positioned in the trim adjacent to the rear seats. Measurements of the various plant responses and the spectral properties of the microphone signals have been conducted using a sampling frequency of $2.56 \mathrm{kHz}$. These measurements are used below to calculate the optimum feedback and feedforward controllers and hence the potential performance of these systems in a real application. In practice an adaptive implementation would converge to these optimal solutions. 

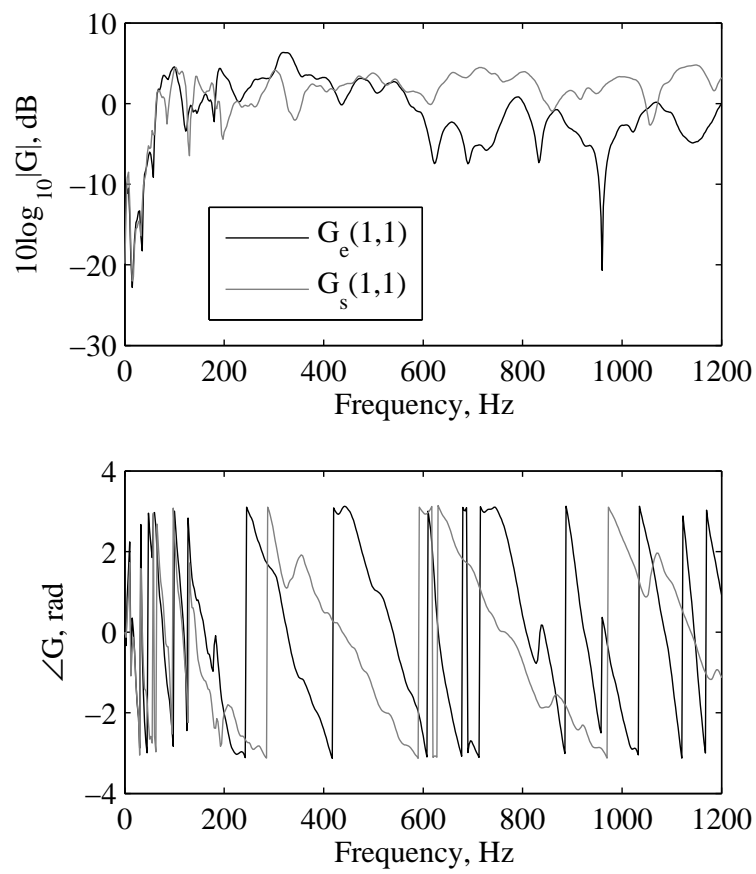

Figure 5: Examples of the plant frequency response, measured between the voltage input to car audio loudspeaker positioned in the driver's door and the pressures produced at the headrest microphone $(-)$ and the floor microphone $(-)$.

\subsection{Car Cabin Environment}

\subsubsection{Measured Plant Responses}

The plant response between the four car audio loudspeakers and the eight microphones has been measured and Figure 5 shows examples of the frequency response between the loudspeaker positioned in the driver's door and the error microphone positioned at the driver's right ear position and the reference microphone positioned in the driver's foot-well. From this plot it can be seen that a large number of modes contribute to the plant responses and this high order system is well suited to the frequency domain design method employed in the previous sections.

To help test the suitability of the multiplicative output uncertainty model, the plant response has also been measured under three different occupancy conditions. Figure 6 shows the response between the loudspeaker positioned in the driver's door and the error microphone positioned at the driver's right ear position for these three different conditions. From these results it can be seen that at frequencies below $190 \mathrm{~Hz}$ there is minimal variation in the measured responses, while at higher frequencies there are some significant variations in both the magnitude and phase responses. The effects of these variations on the MIMO controllers will be considered below.

\subsubsection{Road Noise Disturbance and Reference Signals}

The pressure produced at the eight microphone positions has been measured when the car is driven under a number of conditions. Figure 7 shows the power spectral densities calculated from the pressure signals measured by the microphone at the driver's right ear position, when the small city car is driven 

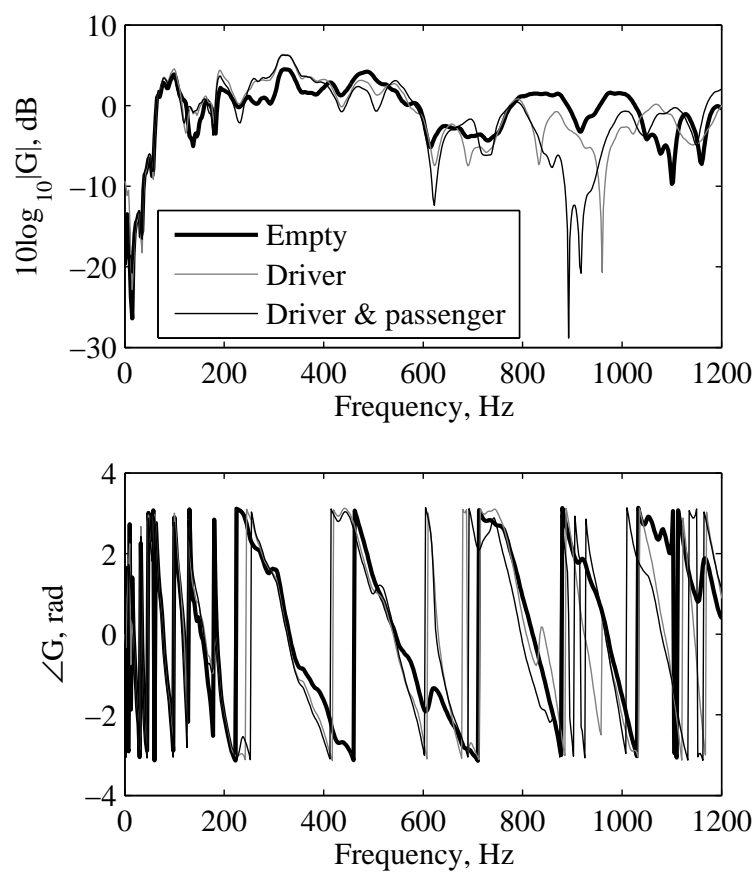

Figure 6: The plant response measured between the voltage input to car audio loudspeaker positioned in the driver's door and the pressure produced at the headrest microphone when the car cabin is empty, occupied by the driver alone and occupied by driver and front passenger.

under four different conditions. From these results it can be seen that in all cases the disturbance is dominated by frequencies below around $300 \mathrm{~Hz}$. It can also be seen that there is a particular problem between around 80 and $200 \mathrm{~Hz}$ when the car is driven over the pave road surface. Such low frequency noise may become even more of a problem in the future, when the weight of vehicles is pushed further down to improve fuel efficiency.

In order for a feedforward control system to achieve control it is necessary for the reference signals to be correlated with the error signals, since the cancellation signals are derived directly from the reference signals, as is clear from equation 18. The ability of the chosen reference and error sensors to fulfill this requirement can be measured by calculating the multiple coherence between the reference signals and each of the headrest error microphones [5]. Figure 8 shows the multiple coherence between the disturbance and reference signals measured when the car is driven at a constant speed of $50 \mathrm{~km} / \mathrm{h}$ over the pave road surface for the cases when (a) all four floor positioned microphones are used as reference signals and (b) only the two front floor positioned microphones are used as reference signals. From these results it can be seen that the four reference sensors are strongly coherent with each of the four error sensor signals at frequencies up to around $180 \mathrm{~Hz}$. At higher frequencies there is a loss of coherence, which indicates that the four reference microphones do not detect all of the noise reaching the error microphones and, therefore, the achievable levels of control will be limited. For the case when only two reference signals are used, the reference signals are only coherent with the disturbance signals at very low frequencies. Therefore, if only 2 reference microphones were employed, the achievable control would be very limited, which highlights the need to use multiple reference signals in order to facilitate control. It is important to highlight that the drop-off in coherence between the reference and error signals is not 


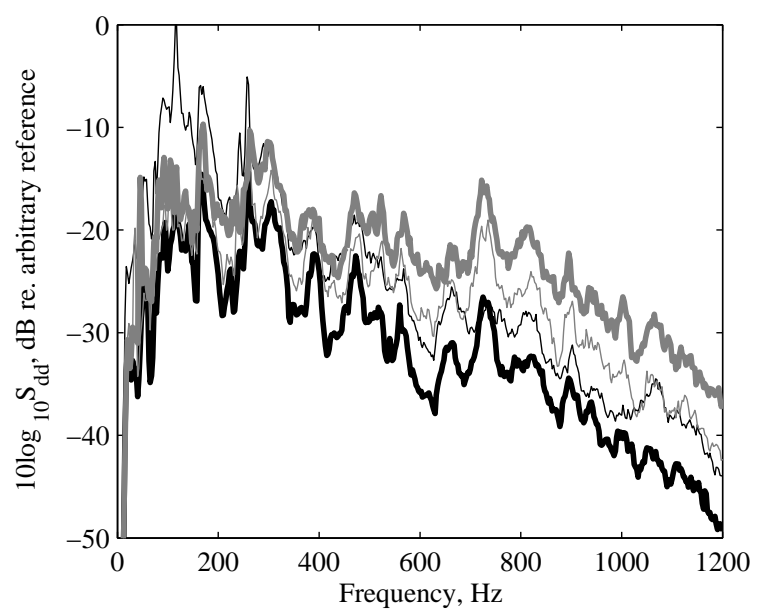

Figure 7: The A-weigted power spectral density of the pressure measured at the driver's right ear error microphone, $S_{d_{1} d_{1}},(-)$ when the car was driven at $50 \mathrm{~km} / \mathrm{h}$ over a pave road surface (thin black line), and at $50 \mathrm{~km} / \mathrm{h}$ (thick black line), $75 \mathrm{~km} / \mathrm{h}$ (thin grey line) and $100 \mathrm{~km} / \mathrm{h}$ (thick grey line) over a rough road surface.

due to the use of low-cost microphones, which in fact have a bandwidth of $20 \mathrm{~Hz}$ to $16 \mathrm{kHz}$, but are due to the spatial variance of the sound field within the car cabin.

The level of achievable attenuation can be related to the multiple coherence function for a controller with no causality constraints as [5]

$$
\operatorname{Attn}=-10 \log _{10}\left[1-\gamma^{2}\right]
$$

where $\gamma^{2}$ is the multiple coherence function. From this relationship, for a multiple coherence between the reference signals and disturbance signals of 0.9 , the maximum achievable attenuation will be limited to $10 \mathrm{~dB}$. Based on the acoustic limitations of active noise control in an enclosure [27], the achievable control at higher frequencies will also be limited by the increasing number of excited acoustic modes and the number of control sources practically available, and in view of these limitations, the drop in coherence at high frequencies is not necessarily a severe problem.

\subsection{Active Noise Control Performance}

\subsubsection{Nominal Performance}

The aim of both of the multichannel road noise controllers is to minimise the sum of the squared pressures at the headrest microphone positions, whilst maintaining the relevant robustness, disturbance enhancement and open-loop controller stability constraints as detailed in Section 2. This optimization process has been implemented for the feedback and feedforward control systems using the methods described above to achieve the objectives described by equations 26 and 27 respectively. In each case the plant response matrices, $\mathbf{G}(k)$, have been formed from the frequency responses measured in the small city car, between the four loudspeakers and the four headrest error microphones and the four floor reference microphones, for a nominal plant condition, with only the driver in the vehicle. The disturbance and reference signals used to calculate the spectral density matrices in equations 26 and 27 are those measured when the car is driven at $50 \mathrm{~km} / \mathrm{h}$ over a pave road surface. The plant responses and spectral 


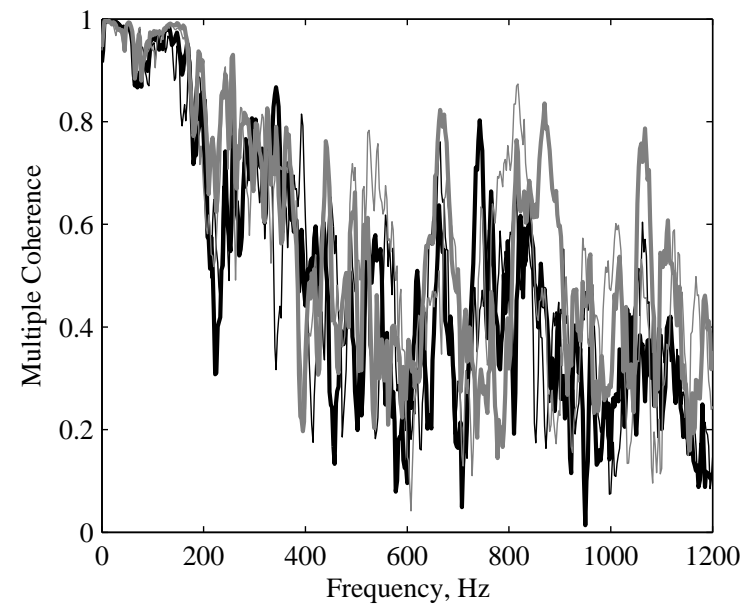

(a) All four reference microphones.

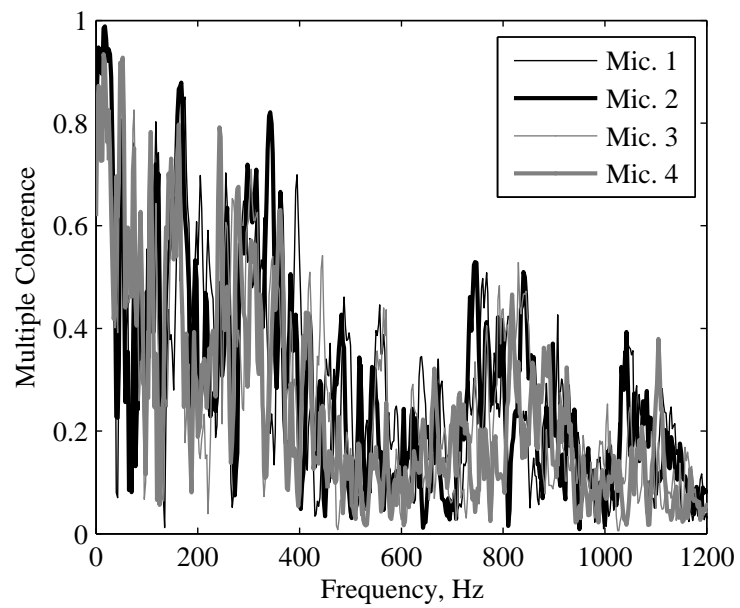

(b) Front two reference microhpones.

Figure 8: The multiple coherence between each of the headrest microphone signals and four or two of the reference microphone signals, measured when the car was driven at $50 \mathrm{~km} / \mathrm{h}$ over a pave road surface. 
densities obtained from the car cabin measurements have been discretised at $N=513$ frequencies, which provides sufficient accuracy so that the corresponding impulse responses or correlation functions have negligible amplitude at the end of their responses.

The enhancement constraint, which is identical for both control systems, has been defined as $A=4$, which ensures a maximum enhancement in the individual headrest error signals of $6 \mathrm{~dB}$. The bound on the output uncertainty has been set to $B=0.5$ for both control systems. This ensures that equations 13 and 24 are satisfied at all frequencies for the plant responses measured under the three different occupancy conditions. It is worth highlighting that both the robust stability and open-loop stability constraints for the feedback and feedforward controllers are different, since the feedforward stability constraints only consider the plant responses relating to the reference sensors located on the floor of the car cabin and contained in the matrix, $\mathbf{G}_{s}$, whereas the feedback stability constraints consider the full matrix of plant responses between the car cabin loudspeakers and both the headrest and floor positioned microphones, $\mathbf{G}=\left[\mathbf{G}_{e}^{T}, \mathbf{G}_{s}^{T}\right]^{T}$.

To calculate the solutions of equations 26 and 27 the control filters have been defined as $I=128$ coefficient FIR filters, which for the feedforward controller with $K=4$ reference sensors and $M=4$ control sources gives a total of 2048 coefficients to be optimised and for the feedback controller with $L=L_{e}+K=8$ feedback signals and $M=4$ control sources gives a total of 4096 coefficients to be optimised. The suitability of this filter length has been validated for both control strategies by increasing the filter length and ensuring that no significant improvement in control is achieved.

Figure 9 shows the robust stability constraint, the enhancement constraint and the open-loop stability constraint for the feedforward (black lines) and feedback (grey lines) controllers, plotted against frequency up to the Nyquist frequency. The first subplot in Figure 9 shows the robustness constraint for the feedback and feedforward controllers given by equations 13 and 24 respectively. In both cases the constraint is satisfied provided that the constraint function is less than unity and it can be seen that this is achieved for both controllers up to the Nyquist frequency. It can be seen that the two controller show a similar level of robustness according to this metric and the suitability of this robustness constraint to practical variations in the plant responses will be investigated further below.

The second subplot in Figure 9 shows the disturbance enhancement constraint given by equation 14, which is also satisfied since the plotted function is less than unity. From the presented results in Figure 9 it can be seen that this constraint is also satisfied by both controllers. However, to provide more insight into the novel disturbance enhancement constraint described by equation 14, Figure 10 shows the maximum enhancement in any of the headrest error signals, that is $\max \left[\operatorname{diag}\left(\mathbf{D}(j \omega) E\left(\mathbf{e}_{e}(j \omega) \mathbf{e}_{e}^{H}(j \omega)\right)\right)\right]$, against frequency. From this plot it can be seen that the maximum enhancement in the individual pressures for both the feedforward and feedback controllers is below $6 \mathrm{~dB}$ at all frequencies although the maximum enhancement for both controllers occurs at around $360 \mathrm{~Hz}$ and the constraint is active at this frequency. This indicates that the proposed enhancement constraint has been effective and ensures that the enhancements in the individual errors are limited to be below the defined value of $6 \mathrm{~dB}$, corresponding to $A=4$.

The final subplot in Figure 9 shows the constraint functions related to the stability of the open-loop controller, which are by equations 16 and 25 for the feedback and feedforward controllers respectively. These constraint functions are once again satisfied provided that the their values are less than unity and it can be seen that this is achieved across the full bandwidth of the controller. To provide further insight 

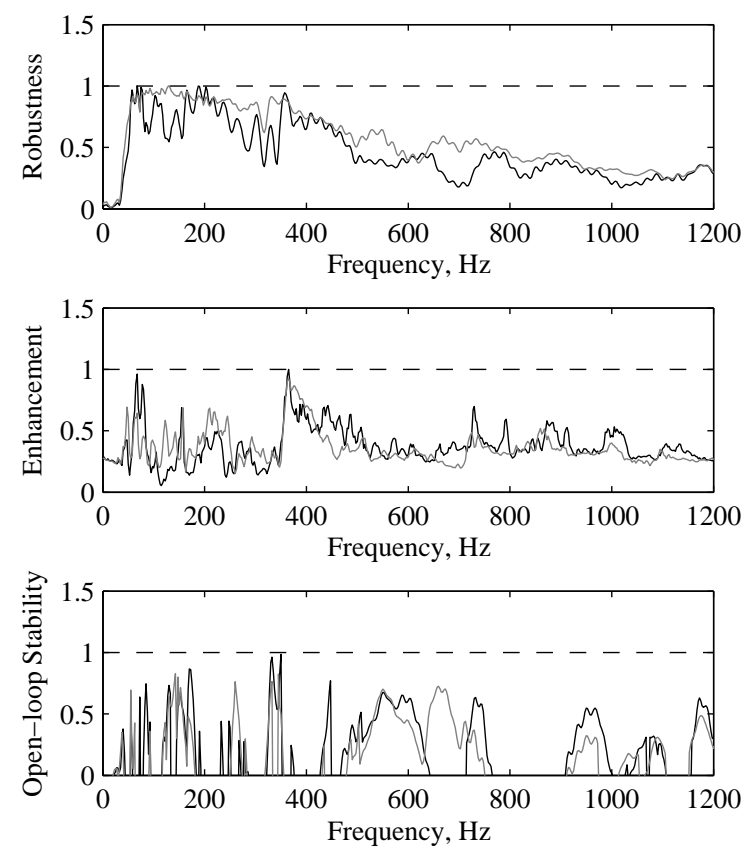

Figure 9: Robust stability, disturbance enhancement and open-loop stability constraints for the optimised feedforward (-) and feedback $(-)$ controllers. Each constraint must be less than unity (indicated by the dashed lines) at all frequencies for the constraints to be satisfied

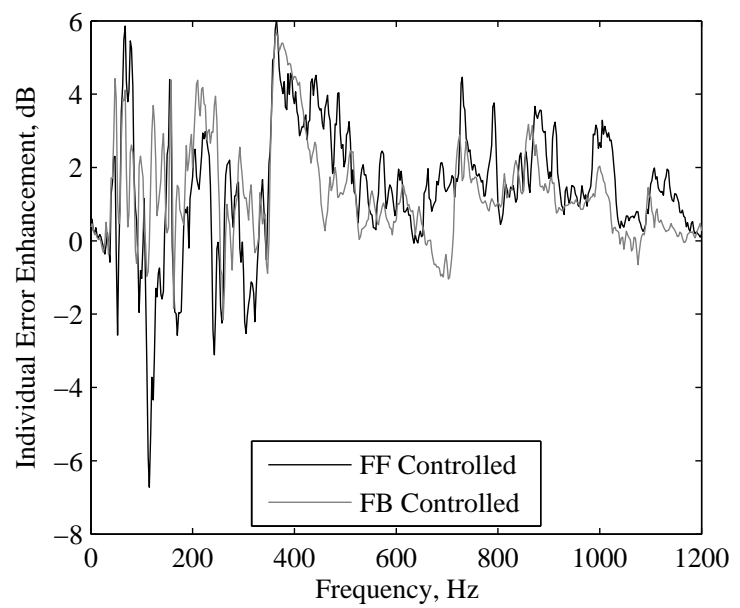

Figure 10: The maximum enhancement in the individual error signals for the feedforward $(-)$ and feedback (-) controllers. 


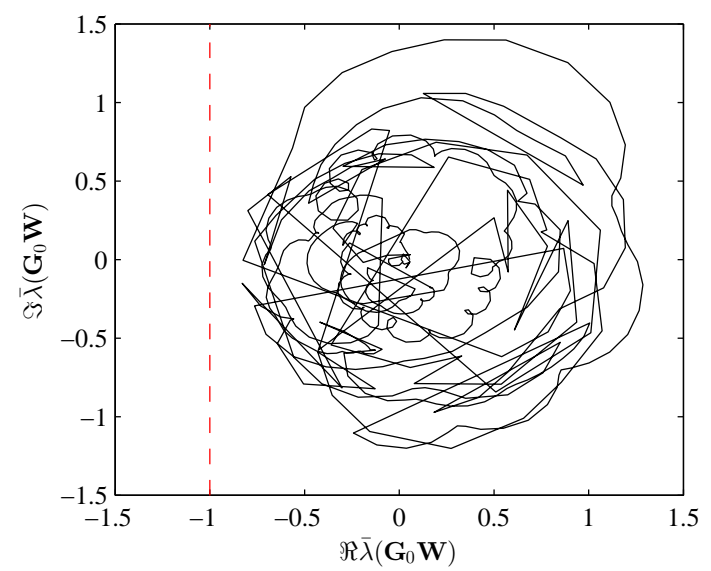

(a) Feedback controller.

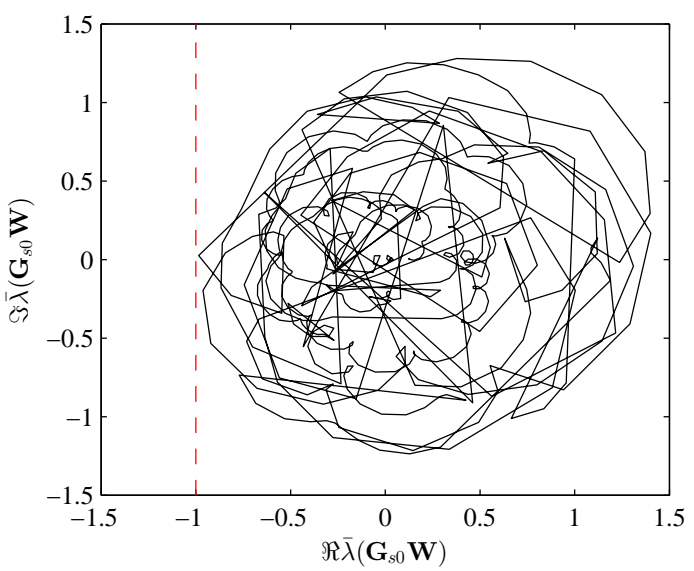

(b) Feedforward controller.

Figure 11: The Nyquist plots demonstrating the open-loop controller stability constraint for the feedback (a) and feedforward (b) controllers. The constraint limit is also shown by the dotted line.

into the open-loop stability constant, Figure 11 shows the Nyquist plots of the maximum eigenvalues of the open-loop responses for the two controllers. From these plots it can be seen that both of the controllers are open-loop stable, although the feedforward controller is marginally closer to instability.

The nominal performance of the two optimised controllers has been calculated, according to the cost function given by the sum of the squared pressures measured at the headrest error microphones, using the nominal plant frequency responses and the road noise signals measured at the eight microphones when the car is driven at $50 \mathrm{~km} / \mathrm{h}$ on a pave road surface. The uncontrolled and controlled results are presented in Figure 12 up to $500 \mathrm{~Hz}$, since at higher frequencies there is negligible variation between the controlled and uncontrolled results. From these results it can be seen that the broadband peak in the disturbance spectrum between 80 and $200 \mathrm{~Hz}$ has been attenuated to a similar level by the two controllers, with a maximum reduction at $115 \mathrm{~Hz}$ of $8 \mathrm{~dB}$ and an average reduction of around $3 \mathrm{~dB}$. At higher frequencies the differences in performance between the two controllers become more significant. For example, although both controllers achieve $4 \mathrm{~dB}$ attenuation at the $240 \mathrm{~Hz}$ peak, which is due to the tyre cavity resonance, the feedback controller achieves $3 \mathrm{~dB}$ more attenuation at the $260 \mathrm{~Hz}$ tyre cavity resonance than the feedforward controller. At frequencies between 260 and $350 \mathrm{~Hz}$ the feedback controller continues to achieve higher levels of attenuation than the feedforward controller.

The differences in performance between the feedback and feedforward controllers shown in Figure 12 can be related to the signals available to the two control filters. That is, while the feedback control filter is driven by all eight microphone signals, with four positioned at the headrests and four positioned on the floor of the cabin, the feedforward control filter is only driven by the four reference signals from the microphones positioned on the floor of the car cabin and, therefore, the achievable control is dependent on the level of correlation between these four reference signals and the pressures at the headrest microphones. As discussed above in reference to Figure 8, the multiple coherence between the reference signals and the four headrest error signals begins to roll-off at frequencies above around $180 \mathrm{~Hz}$ and, therefore, the feedforward controller is no longer able to control the pressures produced at the headrest error microphones, in contrast, the feedback controller uses the headrest error signals directly and, therefore, is not limited by this constraint. 


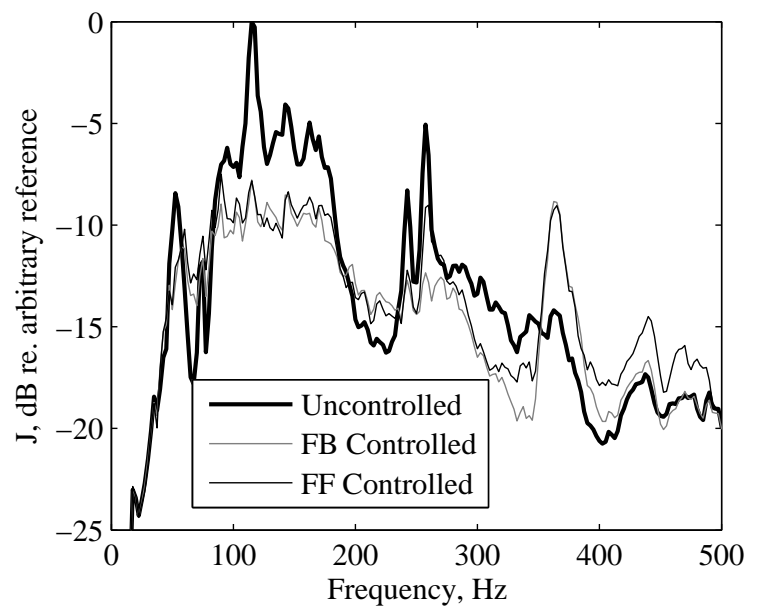

Figure 12: The sum of the squared pressures measured by the headrest error microphones before control $(-)$ and after control using the optimised feedforward $(-)$ and feedback $(-)$ controllers. The results are plotted in decibels with respect to an arbitrary reference level.

\subsubsection{Robustness to Plant Variations}

Although significant levels of control have been predicted for the nominal plant response condition, in practice the plant response will vary over time and, therefore, the modelled plant responses used in the feedback and feedforward controllers, as shown in Figures $1 \mathrm{~b}$ and 3b, will not perfectly match the actual plant responses. The controller designs presented have included a robust stability constraint that is designed to allow the controllers to remain stable in the face of changes in the plant responses, however, it is important to also evaluate the control performance under these conditions. Figure 13 shows the cost function calculated for the feedback controller, with the disturbance signals measured when the car is driven at $50 \mathrm{~km} / \mathrm{h}$ over the pave road surface, for the nominal plant response, measured with only the driver in the car, and when the physical plant response is that measured with an empty car cabin, or when the car cabin is measured with two front seat occupants; in this instance the results are shown over the full bandwidth of the controller to highlight that the enforced robustness constraint is sufficient. Although the individual controlled responses cannot be identified from this plot, it is clear that these practical levels of plant variation do not significantly affect the levels of achievable control. Figure 14 shows the equivalent results for the feedforward controller and, once again, it can be seen that the changes in the physical plant response do not significantly affect the performance of the controller.

\subsubsection{The Effect of Disturbance Variation on Performance}

Although it has been predicted that reduction of the broadband peak, which occurs between 80 and $200 \mathrm{~Hz}$ when the car is driven over a pave road surface, is achievable using both the feedback and feedforward control systems, it is interesting to consider how the controller behaves under different road surface conditions and driving speeds. The predicted performance of the two controllers is presented for the three different road conditions in Figure 15, when the fixed feedback and feedforward controllers, optimised for the pave road surface, have been used to predict the attenuation for three further road noise conditions. From the uncontrolled responses in each of these plots, it is clear that the broadband peak between 80 and $200 \mathrm{~Hz}$ observed on the pave road surface is no longer a particular problem. However, 


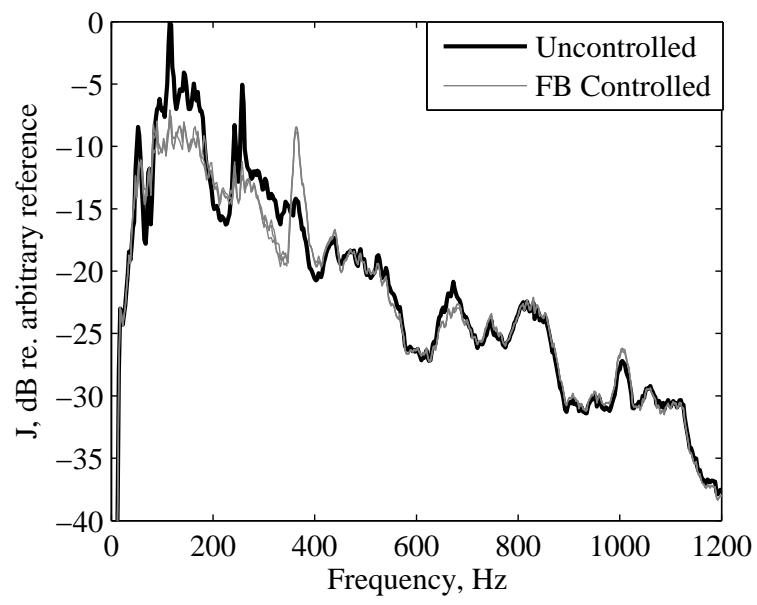

Figure 13: The sum of the squared pressures measured by the headrest error microphones before control $(-)$ and after control using the optimised feedback controller $(-)$ for three different plant responses plotted over the entire frequency range up to half the sampling frequency. The results are plotted in decibels with respect to an arbitrary reference level.

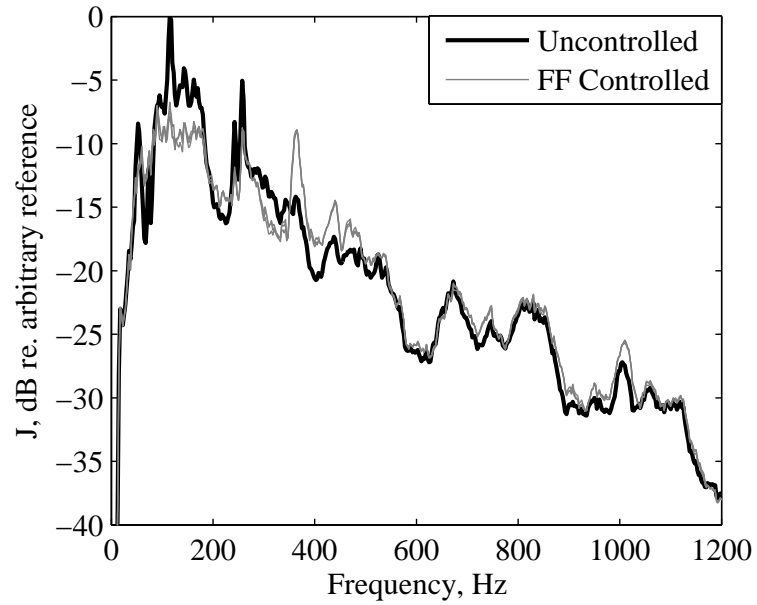

Figure 14: The sum of the squared pressures measured by the headrest error microphones before control $(-)$ and after control using the optimised feedforward controller $(-)$ for three different plant responses plotted over the entire frequency range up to half the sampling frequency. The results are plotted in decibels with respect to an arbitrary reference level. 


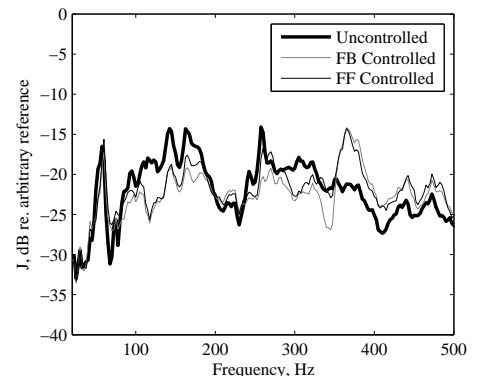

(a) Rough road at $50 \mathrm{~km} / \mathrm{h}$.

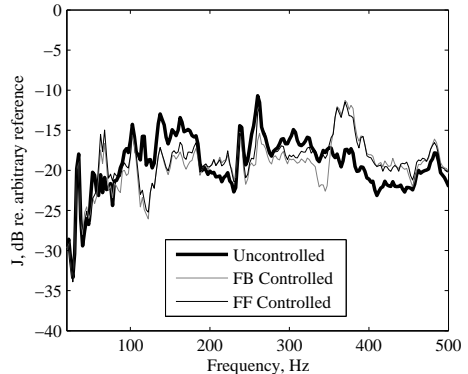

(b) Rough road at $75 \mathrm{~km} / \mathrm{h}$

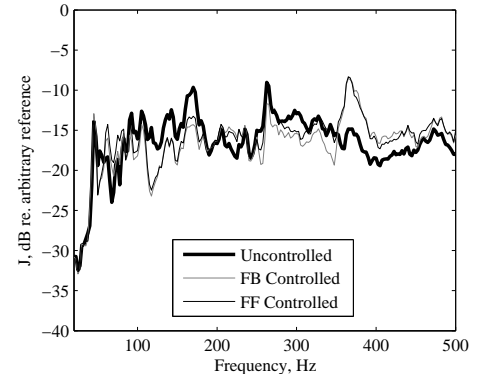

(c) Rough road at $100 \mathrm{~km} / \mathrm{h}$.

Figure 15: The sum of the squared pressures measured by the headrest error microphones before control $(-)$ and after control using the feedforward $(-)$ and feedback $(-)$ controllers optimised for the pave road surface. The results are plotted in decibels with respect to an arbitrary reference level.

it can be seen that for each of these different road conditions some control is still achieved, and more importantly the overall broadband level is not significantly enhanced.

\subsubsection{The Effect of Delays on Performance}

To practically implement the control strategies described here, it would almost certainly be necessary to employ a digital system, due to the number of filters required and their complexity. Although the predictions presented up to this point have considered the delay due to the acoustic propagation and transducer responses, they have not included any additional delay introduced by the digital-to-analogue and analogue-to-digital converters and the required anti-aliasing and reconstruction filters. The effect of delays on the performance of single-input-single-output feedforward and feedback controllers for road noise reduction has been investigated in [14] and it was shown that the performance of the feedback controller was more significantly degraded by delays than the feedforward controller. Therefore, it is important to consider the effect of additional delays on the performance of the multichannel road noise control systems presented here.

To evaluate the effect of additional delays on the performance of the multichannel feedforward and feedback controllers, an additional $2 \mathrm{~ms}$ of delay, which corresponds to 5 samples at the employed sample rate, has been introduced into the control system response and the optimal feedback and feedforward control filters have been recalculated. The resulting performance of the two controllers, for the disturbance due to the pave road surface, is presented in Figure 16. By comparing these results with those in Figure 12 it can be seen that the performance of both the controllers has been reduced over the 80 to $200 \mathrm{~Hz}$ bandwidth by the presence of the additional delay, however, the performance of the feedback controller has been more significantly reduced.

The change in the control performance for the feedback and feedforward controllers due to the additional delay can be related to the operation of the control filters in the two systems. In the feedback system, the control filter, $\mathbf{W}$, filters both the floor and headrest microphone signals to cancel the pressures at the headrest microphones. When there is no additional delay in the system, the feedback controller is able to use the headrest microphone signals to improve the bandwidth of control compared to the feedforward controller, since these signals are inherently coherent with themselves and the associated plant delay is small compared to the variation in the disturbance signals. However, when plant delay is increased, the feedback controller is no longer able to effectively utilise the headrest microphones because 


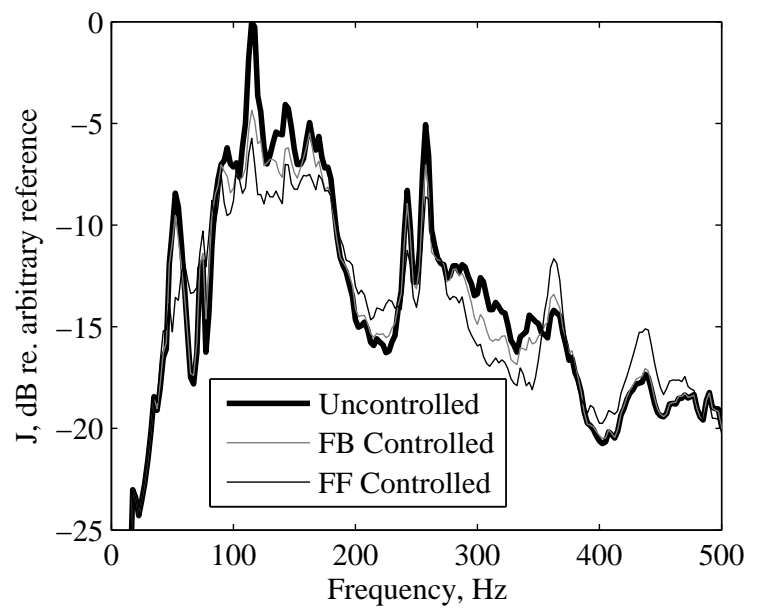

Figure 16: The sum of the squared pressures measured by the headrest error microphones before control (-) and after control using the optimised feedforward $(-)$ and feedback $(-)$ controllers with an additional $2 \mathrm{~ms}$ delay. The results are plotted in decibels with respect to an arbitrary reference level.

the plant delay is no longer small compared to the correlation time of the disturbance signals. In the feedforward case the control filter, $\mathbf{W}_{x}$, filters the floor microphone signals to cancel the pressures at the headrest microphones and the performance is initially limited by the multiple coherence between the reference and error microphone signals. However, in this case, only when the delay is increased to such an extent that the reference sensors no longer provide the control filter with a time advanced signal coherent with the error signals, is the performance of the feedforward controller reduced, and this limitation does not occur until the delay is significantly greater than $2 \mathrm{~ms}$.

\section{Conclusions}

Active noise control is a potentially complementary technology to passive noise control for lightweight vehicles although its application to road noise control has been largely limited by cost. This paper has investigated the potential of implementing a multichannel road noise control system employing low-cost microphones and the car audio loudspeakers; thus avoiding the need for the expensive accelerometers mounted to the vehicle structure as employed in previous road noise control systems [5]. It has been shown that this system can be formulated as either a feedback controller, employing an internal model control architecture, or a feedforward controller with feedback cancellation. These two controller formulations are compared in the context of the road noise control problem, and the results highlight the potential benefits of the two architectures for the implementation of road noise control.

The multichannel feedforward road noise control system employs headrest error microphones, reference microphones on the floor of the car cabin and the car audio loudspeakers. The multichannel feedback formulation of this system, however, employs both the headrest and floor microphones as error signals and thus all of the microphone signals are fed to the control filter. In both systems the controller aims to minimise the sum of the squared pressures measured by the headrest microphones, however, this optimisation process may lead to significant enhancements outside of the control bandwidth and significant enhancements at individual error sensors. To overcome this problem a novel constraint on the enhancement in the individual pressures measured at the headrests has been developed. This new 
constraint on the maximum enhancement is important in practice since the subjective impression of the active control system in a vehicle will be compromised if the sound is significantly increased at one location in order to achieve modest attenuations overall.

The proposed multichannel controllers have been designed using a constrained optimisation technique and by employing FIR control filters, a method of solving this problem in the discrete frequency domain has been presented. Using this design method with plant responses and disturbance signals measured in a small city car, the performance of the feedforward and feedback controllers has been predicted offline. Both systems achieve reductions of up to $8 \mathrm{~dB}$ at low frequencies and an average of about 3 $\mathrm{dB}$ reduction in the sum of the mean squared pressures from 80 to $200 \mathrm{~Hz}$, where there is a particular road noise problem. This level of road noise reduction is comparable to that achieved with previous feedforward road noise control systems employing accelerometer reference signals [5] and, therefore, this highlights the potential to utilise microphone reference signals in active road noise control systems and thus reduce the cost of implementation. Good performance of the feedback controller is seen over a significantly larger bandwidth compared to the feedforward controller, because the performance of the feedforward controller is limited by the low multiple coherence between the reference signals and the error signals at frequencies above around $200 \mathrm{~Hz}$, which is the same limitation in the previous road noise control system investigated in [5]. The effect of plant variations on the two controllers has been simulated using plant responses measured with different numbers of car cabin occupants and it has been shown that the performance of both controllers is not significantly affected by such variations. The effect of the fixed controllers on road noise disturbances produced by different road and driving conditions has also been investigated and, although the targeted road noise problem does not occur under these other driving conditions, the control systems do not significantly enhance the broadband noise level. Finally, the effect of delays in the plant response on the optimised performance of the two controllers has been investigated. This is an important practical consideration due to the inherent delays in a digital implementation. It has been shown that the performance of both controllers has been reduced by the introduction of a $2 \mathrm{~ms}$ delay, however, the performance of the feedback controller is more significantly reduced as expected.

\section{Acknowledgment}

This work was supported by the Green City Car Project, part of the 7th Framework Programme (FP7).

\section{References}

[1] S. J. Elliott, "Active noise and vibration control in vehicles," in Vehicle Noise and Vibration Refinement (X. Wang, ed.), pp. 235-251, Cambridge: Woodhead Publishing, 2010.

[2] S. J. Elliott, I. M. Stothers, P. Nelson, M. A. McDonald, D. C. Quinn, and T. J. Saunders, "The active control of engine noise inside cars," in Proceedings of INTER-NOISE 88 (M. Bockhoff, ed.), vol. 2, (Poughkeepsie, New York), pp. 987-990, 1988.

[3] R. Schirmacher, R. Kunkel, and B. M., "Active noise control for the 4.0 TFSI with cylinder on demand technology in Audi's S-series," Society of Automotive Engineers, no. 2012-01-1533, 2012. 
[4] Y. Kobayashi, T. Inoue, H. Sano, A. Takahashi, and K. Sakamoto, "Active sound control in automobiles," in Proc. Inter-noise, 2008.

[5] T. J. Sutton, S. J. Elliott, M. A. McDonald, and T. J. Saunders, "Active control of road noise inside vehicles," Journal of Noise Control Engineering, vol. 42, pp. 137-146, Jul-Aug 1994.

[6] R. Bernhard, "Active control of road noise inside automobiles," in Proceedings of ACTIVE 95, pp. 21$32,1995$.

[7] S.-H. Oh, H. suk Kim, and Y. Park, "Active control of road booming noise in automotive interiors," Journal of the Acoustical Society of America, vol. 111, no. 1, pp. 180-188, 2002.

[8] J. I. Mohammad, S. J. Elliott, and A. Mackay, "The performance of active control of random noise in cars," Journal of the Acoustical Society of America, vol. 123, no. 4, pp. 1838-1841, 2008.

[9] H. Sano, T. Inoue, A. Takahashi, K. Terai, and Y. Nakamura, "Active control system for lowfrequency road noise combined with an audio system," IEEE Transactions on Speech and Audio Processing, vol. 9, pp. 775-763, October 2001.

[10] J. Cheer and S. J. Elliott, "Spatial and temporal filtering for feedback control of road noise in a car," in Proceedings of the 19th International Congress on Sound and Vibration, (Vilnius, Lithuania), July 2012 .

[11] J. Cheer, Active Control of the Acoustic Environment in an Automobile Cabin. PhD thesis, University of Southampton, Southampton, UK, October 2012.

[12] J. Cheer and S. J. Elliott, "The design and performance of feedback controllers for the attenuation of road noise in vehicles," International Journal of Acoustics and Vibration, Accepted 2013.

[13] J. Cheer and S. J. Elliott, "Multichannel feedback control of interior road noise", in Proceedings of Meetings on Acoustics, vol. 19, 2013.

[14] S. J. Elliott and T. Sutton, "Performance of feedforward and feedback systems for active control," IEEE Transactions on Speech and Audio Processing, vol. 4, pp. 214-223, May 1996.

[15] S. Skogestad and I. Postlethwaite, Multivariable feedback control, analysis and design. Wiley, 1996.

[16] B. Rafaely and S. J. Elliott, " $H_{2} / H_{\infty}$ active control of sound in a headrest: Design and implementation," IEEE Transactions on Control Systems Technology, vol. 7, pp. 79-84, January 1999.

[17] Morari, M. and Zafiriou, E., Robust process control. Prentice Hall, 1989.

[18] S. J. Elliott, Signal Processing for Active Control. London: Academic Press, 2001.

[19] Haddad, W. M. and Bernstein, D.S., "Robust stabilization with positive real uncertainty: beyond the small gain theorem", Systems $\&$ Control Letters, vol. 17, pp. 191-208, 1991.

[20] Tits, A.L., Balakrishnan, V. and Lee, L., "Robustness under bounded uncertainty with phase information", IEEE Transactions on Automatic Control, vol. 44, no. 1, pp. 50-65, January, 1999. 
[21] Schilp, A.T., Multichannel plant estimate for active control. MSc thesis, University of Southampton, Southampton, UK, 2008.

[22] W.-K. Tseng, B. Rafaely, and S. J. Elliott, "Performance limits and real-time implementation of a virtual microphone active headrest," in Active 2002 (P. Gardonio and B. Rafaely, eds.), vol. 2, pp. 1231-1250, 2002.

[23] S. Boyd and L. Vandenberghe, Convex optimisation. Cambridge: Cambridge University Press, 2004.

[24] P. Titterton and J. Olkin, "A practical method for constrained-optimization controller design: $H_{2}$ or $H_{\infty}$ optimization with multiple $H_{2}$ and/or $H_{\infty}$ constraints," in Proceedings of the 29th Asilomar Conference on Signals, Systems and Computers, vol. 2, pp. 1265 -1269, November 1995.

[25] A. Gonzalez, A. Albiol and S.J. Elliott, "Minimisation of the maximum error signal in active control", IEEE Transactions on Speech and Audio Processing, vol. 6, no. 3, pp. 268-281, May 1998.

[26] Mathworks, "Frequency response of a digital filter - Freqz", [online], Available from:http://www. mathworks.co.uk/help/signal/ref/freqz.html, 2014.

[27] P. Nelson and S. J. Elliott, Active Control of Sound. London: Academic Press, 1992. 